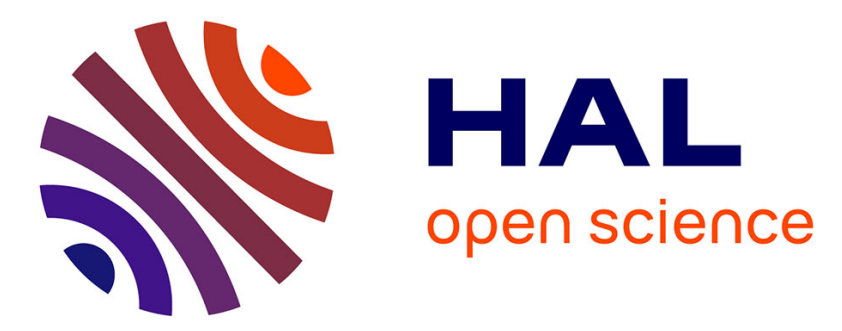

\title{
Modélisation de la dynamique de répartition de l'azote chez un jeune arbre fruitier pendant la phase de croissance exponentielle. I. Etablissement du modèle. Estimation des paramètres
}

Robert Habib, Pascal Monestiez

\section{To cite this version:}

Robert Habib, Pascal Monestiez. Modélisation de la dynamique de répartition de l'azote chez un jeune arbre fruitier pendant la phase de croissance exponentielle. I. Etablissement du modèle. Estimation des paramètres. Agronomie, 1987, 7 (6), pp.401-408. hal-00885007

\section{HAL Id: hal-00885007 https://hal.science/hal-00885007}

Submitted on 1 Jan 1987

HAL is a multi-disciplinary open access archive for the deposit and dissemination of scientific research documents, whether they are published or not. The documents may come from teaching and research institutions in France or abroad, or from public or private research centers.
L'archive ouverte pluridisciplinaire HAL, est destinée au dépôt et à la diffusion de documents scientifiques de niveau recherche, publiés ou non, émanant des établissements d'enseignement et de recherche français ou étrangers, des laboratoires publics ou privés. 


\title{
Modélisation de la dynamique de répartition de l'azote chez un jeune arbre fruitier pendant la phase de croissance exponentielle. I. Etablisse- ment du modèle. Estimation des paramètres
}

\author{
Robert HABIB \& Pascal MONESTIEZ $\left({ }^{*}\right)$ \\ I.N.R.A., Station d'Agronomie \\ (*) Station de Biométrie, Centre de recherches d'Avignon, Domaine St-Paul, F 84140 Montfavet
}

RÉSUMÉ

\begin{abstract}
L'arbre fruitier est analysé comme ćtant un système ouvert représenté par un ensemble de compartiments anatomiques (racines, tronc, rameaux, feuilles) dont les variables descriptives, d'état ou de commande, sont en évolution dynamique. A partir de cette représentation schématique de la réalité, il est proposé un modèle d'organisation de ces compartiments qui permet de rendre compte de la dynamique des transferts d'azote dans l'arbre. Cette dynamique est basće sur une équation régissant les débits d'azote entre les organes végétaux et qui dépend de 2 termes principaux : le premier traduisant la disponibilité de cet azote (teneur en $\mathrm{N}$ de la source) et le second l'intensité de la demande (aciroissement en matière sèche des puits).

Le sous-modèle, correspondant à cette dynamique, comprend 3 paramètres qui sont, chacun, associés à un organe, source d'un débit d'azote. Les valeurs de ces paramètres peuvent être interprétées comme traduisant le caractère plus ou moins important de réserve des compartiments correspondants. L'étude de sensibilité réalisée confirme la nécessité structurelle des 3 paramètres. Son ajustement à un ensemble de données expérimentales conduit à un écart-type résiduel de l'ordre de $0,08 \mathrm{~g}$ de $\mathrm{N}$ pour des masses mesurées variant de 0,1 à $2 \mathrm{~g}$ selon les organes végétaux et les dates de mesures.
\end{abstract}

Mots clés additionnels : Rosaceae, Prunus persica, pêcher, source, puits, réserve azotée

Modelling of the dynamics of nitrogen partitioning in a young fruit tree during the exponential growth stage. I. Modelling and fitting of parameters.

\begin{abstract}
The fruit tree has been analysed as an open system formed of anatomical compartments (roots, trunk, shoots, leaves) whose state or command variables are in dynamic evolution. On the basis of this scheme, a model of compartment organization is proposed which shows up the dynamics of nitrogen transfers in the tree. These dynamics are based on an equation determining the rate of these nitrogen transfers between plant parts. This equation is made up of 2 main variables : one showing the availability of nitrogen at source, which is represented by nitrogen content (DM basis), and the other showing the degree of demand made by the sink, which is represented by the increase in dry matter. The sub-model of nitrogen transfers contains 3 parameters each of which is associated with a plant part, acting as a source of nitrogen. The fit of the parameters can be interpreted as showing the relative importance of the corresponding compartments as nitrogen reserves. A sensitivity study showed the structural necessity of these 3 parameters. The model has been fitted to experimental data. It leads to a mean residual error of $0.08 \mathrm{~g} \mathrm{~N}$ which is to be compared with measured amounts of nitrogen between 0.1 and $2 \mathrm{~g}$, according to anatomical compartments and time.
\end{abstract}

Additional key words : Rosaceae, Prunus persica, peach, source, sink, nitrogen reserve.

\section{INTRODUCTION}

Il existe une littérature relativement abondante traitant des différents aspects à prendre en compte dans la modélisation de l'alimentation minérale des plan- tes ; depuis la modélisation des transferts d'eau et de solutés dans le sol (BrESLER, 1973), jusqu'à des modèles intégrant la compétition entre plantes pour les éléments nutritifs (BALDwIN, 1976). Concernant l'alimentation nitrique, les paramètres édaphiques et biologiques intervenant dans ces modèles semblent être 
bien définis (NyE, 1979 ; BHAT \& NYE, 1980 ; BURNS, 1980 ) et permettent de simuler l'absorption du nitrate de façon satisfaisante (NYE, 1979) pour des conditions expérimentales assurant l'homogénéité du milieu moyennant certaines hypothèses sur la répartition aléatoire du système racinaire et l'existence de conditions de flux permanent (BALDWIN et al., 1973). Cette approche par modélisation a pour but, à terme, de prévoir la synthèse de la biomasse à partir de l'estimation des prélèvements minéraux de la culture (BARNES et al., 1976 ; BALDWIN, 1976 ; BHAT et al., 1979).

Ces études ont toutes porté sur des cultures annuelles (oignon, choux, colza, maïs, ...) pour lesquelles il est raisonnable de penser qu'il existe une relation directe entre les conditions de nutrition et le comportement des plantes en terme de croissance en matière sèche. Vouloir extrapoler directement cette démarche aux arbres fruitiers, y compris pendant la phase juvénile pré-productive, conduirait probablement à des difficultés du fait de l'existence d'un volant de réserves azotées endogènes important (TromP, 1983; TITUS \& KANG, 1982). Cependant, les quelques données disponibles dans la littérature portant sur l'absorption de nitrate par des rosacées fruitières (THERIOS et al., 1979 ; BHAT, 1982) semblent indiquer que les mécanismes en sont comparables et peuvent être traités mathématiquement de façon identique. Une différence importante néanmoins est la capacité des arbres fruitiers à absorber du nitrate en période hivernale en l'absence de surface foliaire, probablement liée à l'existence d'un pool de réserves carbonées important (HABIB, 1984).

On conçoit donc que toute tentative de modélisation de l'élaboration de la biomasse passe (en excluant pour l'instant l'aspect production fruitière), dans le cas des arbres fruitiers, par la prise en compte de la constitution et de la mobilisation des réserves azotées. Cependant, dans un premier temps, les auteurs se proposent de montrer qu'il est possible, en formulant des hypothèses simples sur les relations d'équilibre entre les différents organes d'un arbre, d'établir un modèle qui simule l'absorption et la répartition de l'azote dans la plante, à partir de la connaissance des courbes de croissance en matière sèche des organes végétaux.

Considérer le «produit final » (la biomasse formée) comme une entrée du modèle en tentant de simuler la dynamique de sa constitution, en terme de masse d'azote exportée, c'est prendre le problème à l'inverse du sens où il est généralement posé. Cependant, cette approche présente l'avantage de placer la constitution et la mobilisation des réserves azotées au centre même des mécanismes à retenir pour la modélisation puisque toute prévision de la répartition de l'azote dans l'arbre est liée à la fois aux besoins des organes en croissance et à la disponibilite en azote de réserve.

L'approche envisagée s'appuie sur certains aspects de la théorie des systèmes quant à la formulation du modèle et au vocabulaire utilisé (CHEVALLIER \& LEFEVRE, 1984). Les objectifs a priori de la modélisation sont d'une part d'aboutir à un modèle le plus simple possible (modèle de transfert à compartiments d'un système dynamique en évolution), et d'autre part de baser les équations de transfert sur des hypothèses et des variables physiques (débit de matière, concentration...). Les auteurs se proposent dans cet article d'aborder les deux premières phases de la modélisation :

- caractérisation de la structure du système (aspect qualitatif),

- identification des paramètres du modèle (aspect quantitatif).

Ce travail s'appuiera sur les données expérimentales présentées par ailleurs (HABIB, 1987) dans le cas où l'alimentation nitrique n'était pas limitante. Les résultats des deux autres essais, comportant une limitation de l'absorption de nitrate, seront utilisés pour la phase de validation du modèle (MONESTIEZ \& HABIB, 1987).

\section{ORGANISATION DU MODÈLE}

\section{A. Hypothèses simplificatrices}

Sur le plan anatomique, on considère généralement que les réserves azotées sont surtout localisées dans le système racinaire des arbres fruitiers, mais également dans les autres parties pérennes de l'arbre (TROMP, 1983). Dans le cas de l'expérimentation effectuée, nous avons fait en sorte que, par un élagage sévère des racines et parties aeriennes a la plantation, seul le tronc puisse être considéré comme un organe de réserve au moment de la plantation (HABIB, 1987).

Les réserves azotées se définissent selon leur caractère soluble (principalement les acides aminés) ou insoluble (principalement les protéines) par référence au solvant utilisé pour l'extraction. Cependant, il a fallu simplifier considérablement le modèle biologique sous-jacent au modèle mathématique. Ainsi, on n'a pas distingué les différentes fractions, soluble et insoluble, de l'azote organique, ne considérant que l'azote total. Ceci appelle deux remarques:

- Cette simplification ne peut être acceptée que si la fraction minérale de l'azote (c'est-à-dire $\mathrm{NO}_{3}$ ) est faible vis-à-vis de l'azote total : on a admis que, dès son absorption par le système racinaire, le nitrate était réduit en acides aminés (TITUS \& KANG, 1982).

- L'utilisation de l'azote total comme variable représentative de la répartition des assimilats suppose que la transformation protéines - acides aminés de transfert est immédiate et sous le contrôle des «besoins » de la plante. Cependant, le caractère de plus ou moins grande disponibilité de l'azote dans chacun des compartiments anatomiques de l'arbre peut être, en partie, rendu par la structure topologique du modèle (c'est-à-dire l'organisation des relations entre les différents compartiments).

\section{B. Caractérisation de la structure globale du modèle}

\section{Définition et organisation des sous-modèles}

Le système global peut être décomposé en un ensemble de sous-systèmes ayant des relations entre eux (fig. 1). Cet ensemble peut être analysé de 2 façons :

\section{a) Partition fonctionnelle}

Sur le plan de son fonctionnement, le système « arbre-milieu» peut être décomposé en sous- 


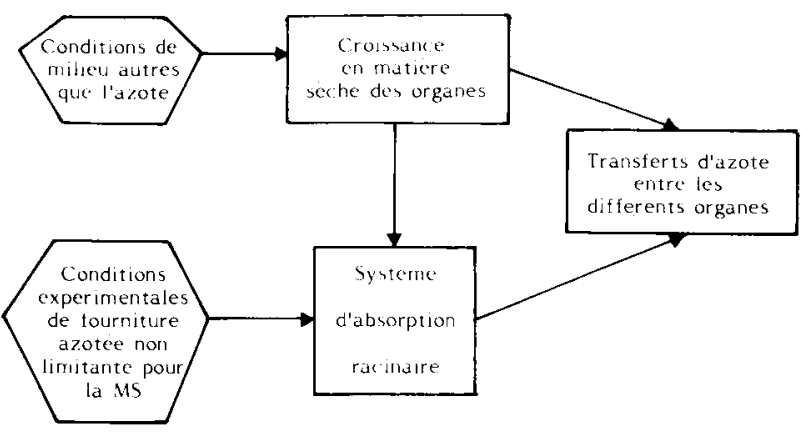

Figure 1

Organisation du système global. Les 3 sous-systèmes représentés par des rectangles sont modélisés. Les flèches représentent les commandes des sous-systèmes entre eux.

Organisation of the global system. The 3 sub-systems represented by rectangles are modelled. The arrows show the commands of the sub-systems between each other.

systèmes ayant des relations entre eux. Les variables d'état d'un sous-système peuvent être les commandes d'un autre. Cette partition ne préjuge pas de la connaissance a priori que l'on a sur le fonctionnement de chacun des sous-systèmes, ni donc sur la nature des sous-modèles utilisés pour leur description (statistique, déterministe, ...), par contre, elle définit le sens des relations que nous imposons entre les soussystèmes, c'est-à-dire l'organisation des commandes. Par exemple, sur la figure 1 on trouve formalisée l'hypothèse développée au paragraphe 1 selon laquelle la répartition de l'azote dans la plante est liée à la connaissance des courbes de croissance en matière sèche des organes végétaux.

\section{b) Partition en compartiments (fig. 2 et 3 )}

Le système " arbre-milieu " peut également être décomposé en compartiments, parties du système que l'on peut isoler et caractériser par des variables (dites variables d'état) dont on modélisera les évolutions dans le temps. Les différents compartiments retenus sont :

- la solution nutritive caractérisée par son volume, sa concentration en azote $\left(\mathrm{NO}_{3}\right)$ et la masse d'azote dissous (fig. 2).

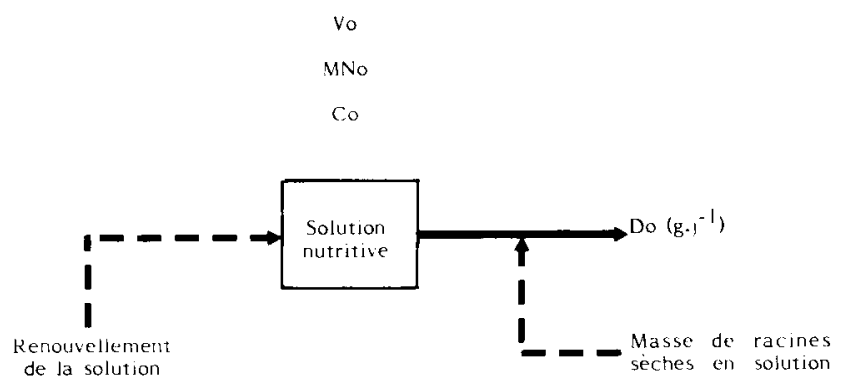

Figure 2

Organisation du sous-système « absorption nitrique » : débit de sortie $D_{o}$ (trait plein) et commandes (tireté), signification des symboles donnés dans le tableau 1.

Organisation of the "nitrate absorption " sub-system : rate of exit $D_{o}$ (continuous line) and commands (dashed line). Symbols given in table 1.

- les organes de l'arbre caractérisés respectivement par une masse de matière sèche, une masse d'azote total et la teneur en $\mathbf{N}$ (p. 100 de MS). Les 4 organes pris en compte sont les racines, le tronc, les rameaux et les feuilles (fig. 3).

Comme cela a été présenté précédemment, notre choix est de prendre le premier sous-système décrivant les croissances en MS des organes de l'arbre comme pilote de l'ensemble. Les variables MS de ce sousmodèle et leurs évolutions seront donc modélisées dans une première étape.

Le second sous-système décrit l'absorption racinaire. Les variables caractéristiques (volume de solution et masse d'azote dissous) subissent 2 types d'influences extérieures (ou commandes) : le calendrier de fourniture azotée (conditions fixées par l'expérimentateur) et la croissance racinaire décrite par le sous-système précédent.

Ces deux derniers sous-systèmes pilotent enfin le sous-système décrivant les transferts d'azote entre organes, objet essentiel de notre étude. Les variables d'état sont les masses d'azote et les teneurs $\left(\mathrm{g} . \mathrm{g}^{-1}\right.$ de MS) de chaque organe.

Les notations utilisées pour les variables ainsi que leurs unités se trouvent rappelées dans le tableau 1. Les compartiments correspondants sont signalés par un indice variant de 0 à 4 qui renvoie respectivement à la solution et aux racines, tronc, rameaux et feuilles.

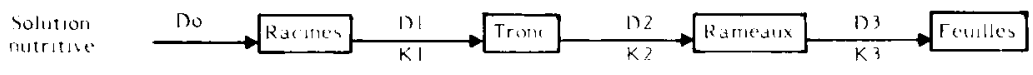

(u)

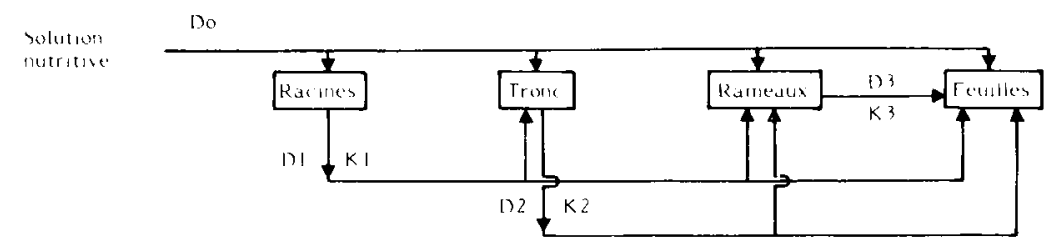

(b)

Figure 3

Structure du modèle de transfert de l'azote: (a) en série, (b) en parallèle.

$D_{j}$ débit d'azote sortant de l'organe " $j$ "

$K_{j}$ paramètre d'ajustement de $D_{j}$

(j varie de 1 à 3 et renvoie respectivement aux racines, tronc et rameaux).
Structure of the nitrogen transfer model : (a) in series, (b) in parallel.

$D_{j}$ is the rate of nitrogen transfer from plant-part « $j$ »

$K_{j}$ is a parameter used in fitting $D_{j}$

(j from 1 to 3 refers respectively to roots, trunk and shoots). 


\section{TABLEAU 1}

Variables caractérisant les compartiments du système * l'indice $i$ varie de $l$ à 4 et renvoie respectivement aux racines, tronc, rameaux et feuilles,

** l'indice $j$ varie de 0 à 3 et renvoie respectivement à la solution et aux racines, tronc, rameaux.

Compartment variables of the system

* i from 1 to 4 refers respectively to roots, trunk, shoots and leaves.

** $j$ from 0 to 3 refers respectively to solution, roots, trunk and shoots.

\begin{tabular}{|c|c|c|}
\hline Variables & Sens & Dimensions \\
\hline \multicolumn{3}{|c|}{ Solution : } \\
\hline Vo & Volume de la solution nutritive & 1 \\
\hline MNo & Masse d'azote $N$ en solution & g \\
\hline Co & Concentration de la solution & g. $1^{-1}$ \\
\hline \multicolumn{3}{|l|}{ Arbre $*$ : } \\
\hline $\mathrm{MS}_{\mathrm{i}}$ & Masse de matière sèche & $\mathrm{g}$ \\
\hline $\mathbf{M N}_{\mathrm{i}}$ & Masse d'azote $\mathrm{N}$ & $\mathrm{g}$ \\
\hline $\mathrm{T}_{\mathrm{i}}$ & Teneur en azote $\mathrm{N}$ rapportée à $\mathrm{MS}$ & $g \cdot g^{-1}$ \\
\hline \multicolumn{3}{|c|}{ Débit de matière ${ }^{* *}$ : } \\
\hline $\mathrm{D}_{\mathrm{j}}$ & $\begin{array}{l}\text { Débit en masse d'azote } \mathrm{N} \\
\text { exportée du compartiment } \mathrm{j}\end{array}$ & $g \cdot j^{1}$ \\
\hline
\end{tabular}

\section{Modélisation de la croissance en matière sèche}

L'analyse des données expérimentales (HABIB, 1987) avait montré que, dans les conditions de culture utilisées, la croissance en matière sèche était indépendante de la disponibilité en nitrate dans la solution nutritive. Ceci justifie une modélisation unique de cette croissance quel que soit le régime de nutrition azotée.

Les 3 organes de néo-formation, racines, rameaux, et feuilles ont une croissance qui répond bien à une forme exponentielle. Cette forme est tout à fait compatible avec la durée de l'expérience mais devra être modifiée pour des modélisations sur une période plus longue (logistique ou autre : LEBRETON \& Millier, 1982).

$$
\text { Pour } \mathrm{i}=1,3,4: \mathrm{MS}_{\mathrm{i}}=\mathrm{a}_{\mathrm{i}} \exp \left(\mathrm{b}_{\mathrm{i}} \mathrm{t}\right) \text {. }
$$

Pour le tronc, une telle forme ne se justifie pas. C'est un organe dont la matière sèche initiale est très importante par rapport à son évolution durant l'expérimentation. Le modèle le plus simple à retenir était une croissance linéaire, mais des expérimentations plus fines nous ont conduit à retenir deux phases de croissance :

- un palier pendant la première phase de développement des autres organes,

- une croissance linéaire ultérieure commençant approximativement au moment de l'arrêt ou du ralentissement de la croissance en élongation des rameaux (date $t_{0}=67$ jours; HABIB, 1987).

$$
\begin{aligned}
& \mathrm{MS}_{2}=\mathrm{a}_{2}^{\prime} \text { si } \mathrm{t}<\mathrm{t}_{0} \\
& \mathrm{MS}_{2}=\mathrm{a}_{2}+\mathrm{b}_{2}\left(\mathrm{t}-\mathrm{t}_{0}\right) \text { si } \mathrm{t}>\mathrm{t}_{0} .
\end{aligned}
$$

L'ajustement des courbes de croissance a été réalisé indépendamment pour chaque organe et sur tous les arbres. Ces courbes ajustées représentent donc la croissance moyenne d'une population statistique mais l'effet arbre entraîne obligatoirement une liaison entre les résidus correspondant à des organes différents. Les résultats de ces ajustements sont donnés dans le tableau 2.

Il aurait été souhaitable, car plus intéressant pour l'analyse des transferts au plan explicatif, d'avoir un modèle matière sèche par arbre, mais celui-ci doit être estimé avec un seul point (mesure destructive) par courbe exponentielle. Nous traiterons donc la suite avec des courbes de croissance identiques pour tous les arbres.

\section{TABLEAU 2}

Paramètres ajustés des modèles de croissance en matière sèche. Fitted parameters of the dry matter models.

\begin{tabular}{lcc}
\hline \multicolumn{1}{c}{ Compartiment } & Paramètre $\mathrm{a}_{\mathrm{i}}$ & Paramètre $\mathrm{b}_{\mathrm{i}}$ \\
\hline Racines & 1,05 & 0,0278 \\
Rameaux & 0,50 & 0,0338 \\
Feuilles & 1,44 & 0,0312 \\
Tronc & 64,7 & - \\
$\mathrm{t} \leqslant \mathrm{t}_{\mathrm{o}}$ & 28,0 & - \\
$\mathrm{t}>\mathrm{t}_{\mathrm{o}}$ & & 0,550 \\
\hline \hline
\end{tabular}

\section{Modélisation de l'absorption nitrique}

Dans le cadre expérimental auquel nous nous restreignons, il est défini un compartiment solution nutritive dont l'entrée correspond aux conditions expérimentales de renouvellement des solutions et la sortie $\mathrm{D}_{0}$ au débit de nitrate pénétrant dans les racines (fig. 2). Pour un même apport de solution nutritive, la variation de la concentration en nitrate est liée à l'absorption racinaire nitrique et à la variation du volumę de solution résultant de l'absorption hydrique. L'absorption du nitrate est représentée par une fonction du type Michaelis-Menten (NYE \& MARRIOT, 1969). L'absorption hydrique moyenne est calculée à partir de l'ajustement des valeurs expérimentales mesurées (HABIB, 1987) à une équation de type hyperbolique. Connaissant la masse de racines présente en solution, la concentration et le volume initiaux, le système des équations (1), (2), (3) (voir tabl. 1) permet de décrire le comportement de ce compartiment :

$$
\begin{gathered}
D_{0}(t)=\frac{D M \times C_{0}(t)}{K+C_{0}(t)} \times M_{1}(t) \\
\frac{d M N_{0}(t)}{d t}=-D_{0}(t) \\
C_{0}(t)=\frac{M_{0}(t)}{V_{0}(t)}
\end{gathered}
$$

$\mathrm{K}$ valeur de la concentration telle que $\mathrm{D}_{0}=\mathrm{DM} / 2\left(\mathrm{~g} . \mathrm{1}^{-1}\right)$

DM débit maximum d'absorption (g. jour $\left.{ }^{-1} \cdot \mathrm{g}^{-1}\right)$

Les paramètres $D M$ et $K$ de (1) ne peuvent être interprétés au sens de la cinétique enzymatique (LASSALES \& THELLIER, 1974). D'ailleurs, les courbes d'absorption racinaire se présentent plutôt sous la forme de plusieurs branches d'hyperboles qui ont parfois été interprétées comme révélant plusieurs mécanismes de transport (GRIGNON \& TOURAINE, 1981). Cependant le formalisme de l'équation (1) semble ren- 
dre compte de la plupart des courbes d'absorption observées (BARNES et al., 1976).

La valeur de $\mathrm{K}$ a été fixée à $10 \mu \mathrm{M}$ de $\mathrm{NO}_{3}^{-}$par référence aux cinétiques globales d'absorption disponibles pour des arbres fruitiers (THERIOS et al., 1979; BHAT, 1982).

L'évolution au cours du temps des débits d'absorption $\mathrm{D}_{0}$ a été déterminée expérimentalement (HABIB, 1987 : fig. 5 b) et montre 2 phases : augmentation puis diminution de la capacité d'absorption racinaire. Cependant, nous ne disposons pas d'hypothèses phénoménologiques nous permettant de relier cette évolution aux variables du système. Nous avons alors considéré le paramètre DM comme constant et égal à la moyenne des valeurs calculées à partir des données expérimentales : $0,038 \mathrm{gN} \mathrm{j}^{-1} \mathrm{~g}^{-1}$. Une part de l'erreur liée au modèle pourra donc être rapportée à cette simplification.

Le système des équations (1), (2), (3) est non linéaire et peut être résolu, après discrétisation implicite, par la méthode de NEWTON-RAPHSON (STOER \& BULIRSCH, 1980).

La prise en compte de la commande du sous-système (calendrier de fourniture nitrique) ne pose pas de difficultés puisqu'elle correspond à une réinitialisation du système d'équations.

\section{Caractérisation de la structure du modèle de trans- fert de l'azote}

Les variables d'état de ce sous-système sont les masses d'azote contenues à chaque instant dans les différents organes et les teneurs en résultant. Les commandes sont d'une part le débit total d'azote $\mathrm{D}_{0}$ entrant dans la plante et d'autre part les matières sèches des organes ou plutôt leurs accroissements qui représentent les besoins potentiels.

Notre objectif est de modéliser la répartition de l'azote dans l'arbre avec des équations simples ayant une signification physique et avec un minimum de paramètres qui soient constants dans le temps.

\section{Equation du débit élémentaire}

Afin de garder une même logique dans tout le modèle et pour ne pas multiplier le nombre de paramètres à ajuster, nous avons défini une forme unique pour le débit élémentaire : le débit qui sera exporté d'un compartiment donné vers les organes supérieurs de l'arbre dépendra :

- de la teneur en azote du compartiment en question (la source) qui traduit la disponibilité de $\mathrm{N}$,

- de la croissance en matière sèche des organes supérieurs de l'arbre (le puits) qui indique l'intensité de la demande.

$$
\mathrm{Di}=\mathrm{Ki} \times \frac{\mathrm{MNi}}{\mathrm{MSi}} \times \sum_{j>i}\left(\frac{\mathrm{dMSj}}{\mathrm{dt}}\right)
$$

ou

$$
\mathrm{Di}=\mathrm{Ki} \times \mathrm{Ti} \times \sum_{\mathrm{i}>\mathrm{i}}\left(\frac{\mathrm{dMSj}}{\mathrm{dt}}\right)
$$

Le coefficient $K_{i}$ (sans dimension) sera à ajuster pour chaque organe. Il sera normalement positif et sera plus ou moins fort selon que l'organe tend à rendre l'azote très disponible ou au contraire le retenir.

L'application de cette forme de débit élémentaire au système complet peut être envisagée de plusieurs façons. Nous décrirons les 2 cas extrêmes : les compartiments étant alimentés en série ou en parallèle.

\section{Modèle en série}

Dans ce cas, nous prenons en compte la disposition des organes les uns par rapport aux autres et faisons l'hypothèse que l'azote est en quelque sorte stocké au niveau de l'organe avant d'être disponible pour le suivant (fig. 3a).

Une teneur en azote trop faible d'un organe peut alors limiter l'approvisionnement de tous les organes supérieurs. Par contre, il n'est pas possible de réduire la fonction puits à la seule croissance de l'organe immédiatement supérieur (qui ne reflète pas la demande en azote) mais il faut rendre le débit proportionnel à la croissance en MS de tous les organes supérieurs.

Ceci est bien illustré par le débit d'azote entre racines et tronc. Dans un $1^{\text {er }}$ temps, la croissance du tronc est nulle, ce qui annulerait ce débit. Il est donc nécessaire de le rendre proportionnel à la croissance cumulée en MS du tronc, des rameaux et des feuilles.

L'absorption racinaire étant fixée par ailleurs, les transferts d'azote sont donc entièrement décrits par trois relations du type précédent, c'est-à-dire trois coefficients $K_{1}, K_{2}, K_{3}$, les fonctions de croissance en matière sèche et par 4 équations de conservation de masse du type :

$$
\frac{\mathrm{dMN}_{\mathrm{i}}}{\mathrm{dt}}=\mathrm{D}_{i-1}-\mathrm{D}_{\mathrm{i}}
$$

i prenant les valeurs de 1 à 4 (tabl. 1) avec $\mathrm{D}_{4}=0$.

\section{Modèle en parallèle}

L'hypothèse un peu contraignante de l'azote stocké à chaque niveau avant d'être disponible pour les niveaux supérieurs nous a amené à une autre forme qui équivaut à prendre en compte un compartiment sève qui distribue l'azote. La modélisation de ce compartiment est délicate (difficulté pour l'isoler et donc le mesurer, pas de temps des mesures trop grand devant ses variations potentielles), et on le simule en autorisant les transferts directs indépendamment de l'état des organes intermédiaires (fig. 3b).

Avec la même forme que précédemment pour l'équation de débit élémentaire, on répartit l'azote transféré dans tous les organes supérieurs au prorata des dMS de chacun.

Les équations de conservation de masse deviennent :

$$
\frac{\mathrm{dMN}_{\mathrm{i}}}{\mathrm{dt}}=\left(\sum_{j<i} \mathrm{D}_{\mathrm{j}} \frac{\mathrm{dMS}_{\mathrm{i}}}{\sum_{\mathrm{k}>\mathrm{j}} \mathrm{dMS}_{\mathrm{k}}}\right)-\mathrm{D}_{\mathrm{i}}
$$

i prenant les valeurs de 1 à $4, j$ prenant les valeurs de 0 à 3 avec $D_{4}=0$.

Le débit d'absorption étant ainsi distribué, les organes peuvent à leur tour servir de réserve et distribuer 
leur propre azote vers l'ensemble des parties supérieures avec la même règle de répartition.

La signification des $K_{i}$ (équ. (4) et (5)), est alors modifiée. Un $\mathrm{K}$ proche de zéro ou nul signifiera que l'organe donneur est neutre dans la répartition de l'azote. Un $\mathrm{K}$ significativement non nul mettra en évidence son rôle de réserve vis-à-vis d'un transport actif vers les parties supérieures. L'ajustement des $K_{i}$ a été réalisé sous contrainte de positivité.

\section{AJUSTEMENT DES PARAMÈTRES DU MODÈLE DE TRANSFERT}

\section{A. Méthodes de résolution et d'ajustement}

Ces 2 modèles sont définis par des équations différentielles caractérisant les débits et les équations de conservation de masse. Les équations sont discrétisées par méthode implicite avec un pas de temps constant (1 journée, valeur qui a été vérifiée comme suffisante). Les conditions initiales sont les premières valeurs expérimentales pour les masses d'azote de chaque organe $(\mathrm{t}=38$ jours $)$.

L'estimation des paramètres $K_{1}, K_{2}, K_{3}$ est réalisée par la méthode des moindres carrés non linéaire, qui autorise la forme différentielle du modèle tout en fournissant une estimation des variances et des liaisons des estimateurs des paramètres. L'algorithme utilisé est celui de Gauss-Marquardt (BACHACOU et al., 1981).

Le critère de minimisation porte sur les masses et non sur les teneurs car il porte simultanément sur les 4 organes qui sont très différents par leurs matières sèches et leurs teneurs. Ceci est cohérent avec les équations de conservation de masse (6) et (7) : une erreur sur un débit massique en sortie d'un organe source prend la même importance en entrée d'un organe puits, quels que soient les organes considérés. Les teneurs serviront cependant à contrôler qualitativement les ajustements (forme et évolution).

\section{B. Résultats des ajustements}

Pour les 2 modèles précédents, l'ensemble des résultats des ajustements est présenté dans le tableau 3. Nous remarquerons tout d'abord un bon ajustement global des deux modèles (valeurs de l'écart-type résiduel faibles et comparables) et que le paramétrage n'entraine pas de fortes corrélations entre estimateurs. Il est normal que les valeurs de $K_{1}, K_{2}$ et $K_{3}$ soient très différentes d'un modèle à l'autre car leurs sens n'est pas le même dans chacun des deux modèles (cf. $\S$ IIC). La figure 4 représentant les courbes ajustées et les points expérimentaux confirme qu'il est impossible de discriminer les deux modèles uniquement sur la base des masses d'azote exportées. La figure 5 donne les valeurs et les courbes correspondantes pour les teneurs. Les ajustements sont globalement moins bons, mais permettent très nettement de préférer le modèle en parallèle essentiellement en fonction des réponses des organes racines et feuilles. Les teneurs amplifient les écarts entre modèle et points expérimen-
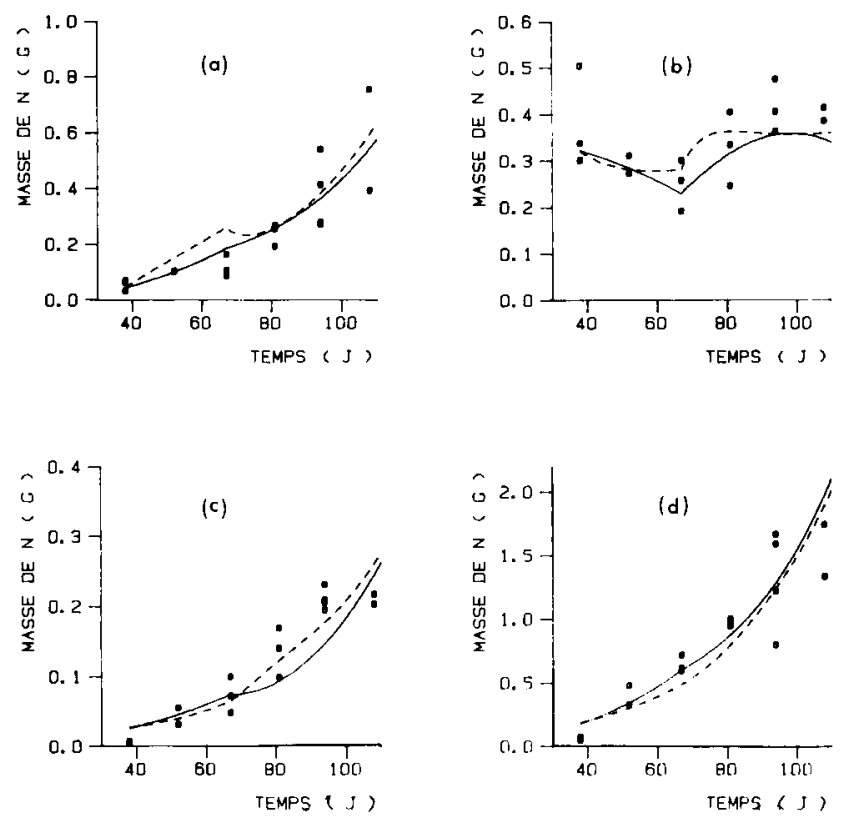

Figure 4

Evolution des masses de $N(g)$ pour les 4 organes: modèle en série (tireté), en parallèle (trait plein) et points expérimentaux. Le premier point des courbes $(t=38 j)$ résulte d'une moyenne de valeurs expérimentales.

(a) Racines, (b) Tronc, (c) Rameaux, (d) Feuilles.

Change in nitrogen amounts (g) for the 4 plant parts: model in series (dashed line), model in parallel (continuous line) and experimental points. The first point on the curve $(t=38 d)$ is taken as the average of experimental values.

(a) Roots, (b) Trunk, (c) Shoots, (d) Leaves.
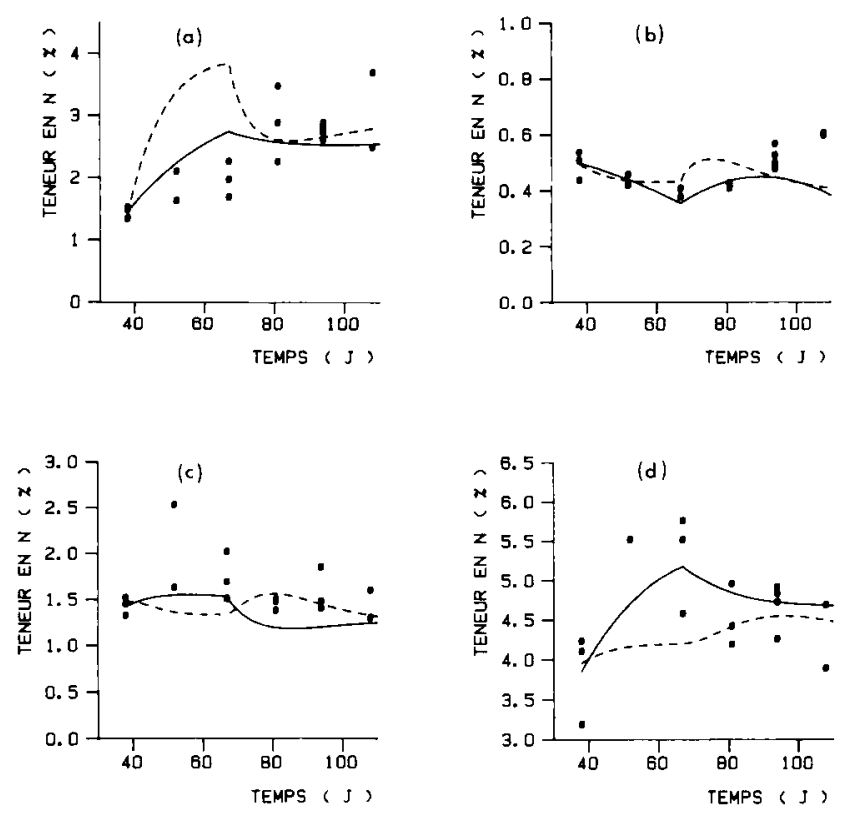

Figure 5

Evolution des teneurs en $N(p .100 \mathrm{MS})$ pour les 4 organes : modele en série (tireté), en parallèle (trait plein) et points expérimentaux. Le premier point des courbes $(t=38 j)$ est une moyenne de valeurs expérimentales.

(a) Racines, (b) Tronc, (c) Rameaux, (d) Heuilles.

Change in nitrogen content (DM basis) for the 4 plant-parts: model in series (dashed line), model in parallel (continuous line) and experimental points. The first point on the curve $(t=38 d)$ is taken as the average of experimental values.

(a) Roots, (b) Trunk, (c) Shoots, (d) Leaves. 
TABLEAU 3

Ajustement des paramètres $K_{i}$ du sous-modèle de transfert de l'azote. L'écart-type initial était de 0,400 $\mathrm{g}$ de $\mathrm{N}$. Fitted Ki parameters of the nitrogen transfer sub-model. The initial standard deviation was $0.400 \mathrm{~g} \mathrm{~N}$.

\begin{tabular}{|c|c|c|c|c|c|c|c|c|}
\hline \multirow{5}{*}{$\begin{array}{c}\begin{array}{c}\text { Modèle } \\
\text { de transfert }\end{array} \\
\text { Série }\end{array}$} & \multirow{5}{*}{$\begin{array}{c}\begin{array}{c}\text { Ecart-type } \\
\text { résiduel } \\
\text { (g de } \mathrm{N})\end{array} \\
0,081\end{array}$} & \multirow{5}{*}{$\begin{array}{l}\mathrm{K}_{\mathrm{i}} \\
\mathrm{K}_{1} \\
\mathrm{~K}_{2} \\
\mathrm{~K}_{3}\end{array}$} & \multirow{5}{*}{$\begin{array}{c}\begin{array}{c}\text { Valeurs } \\
\text { estimées }\end{array} \\
0,90 \\
7,80 \\
3,20\end{array}$} & \multirow{2}{*}{\multicolumn{2}{|c|}{$\begin{array}{l}\text { Limites de } \\
\text { confiance }\end{array}$}} & \multicolumn{3}{|c|}{ Matrice de corrélation } \\
\hline & & & & & & \multirow{4}{*}{$\frac{\mathrm{K}_{1}}{1,0}$} & \multirow{4}{*}{$\frac{\mathrm{K}_{2}}{-\begin{array}{c}0,08 \\
1,0\end{array}}$} & \multirow{4}{*}{$\begin{array}{r}\mathbf{K}_{\mathbf{3}} \\
-0,25 \\
-0,40 \\
1,0\end{array}$} \\
\hline & & & & 0,78 & 1,02 & & & \\
\hline & & & & 6,80 & 8,76 & & & \\
\hline & & & & 2,34 & 4,05 & & & \\
\hline \multirow[t]{3}{*}{ Parallèle } & 0,078 & $K_{1}$ & 0,00 & $-0,03$ & 0,03 & \multirow[t]{3}{*}{1,0} & \multirow{3}{*}{$\begin{array}{c}-0,25 \\
1,0\end{array}$} & $-0,25$ \\
\hline & & $\mathrm{K}_{2}$ & 2,22 & 1,75 & 2,69 & & & $-0,35$ \\
\hline & & $\mathrm{K}_{3}$ & 0,89 & 0,55 & 1,23 & & & 1,0 \\
\hline
\end{tabular}

taux et se révèlent comme un élément indispensable au choix du modèle (les différences observées étaient déjà existantes pour les masses d'azote mais peu significatives).

Pour le modèle en parallèle, les valeurs des coefficients $K_{i}$ permettent la mise en évidence du rôle respectif des comportements de chaque organe dans la répartition de l'azote. La valeur estimée de $K_{1}$ est nulle, ce qui souligne le rôle particulier des racines. ces dernières ne sont pas organe de réserve pendant la phase de croissance exponentielle en MS. $K_{2}$ et $K_{3}$ mettent en évidence le rôle de réserve pris par le tronc et de façon moindre par les rameaux pour fournir un complément d'azote aux feuilles. Il faut cependant remarquer que ces effets ne représentent qu'une loi de comportement général. Les ajustements présentés ici ne permettent pas de conclure à la généralisation au comportement individuel car le suivi arbre par arbre n'a pas été possible.

\section{Sensibilité du modèle aux paramètres}

La phase d'ajustement des paramètres $K_{i}$ permet également d'estimer leurs intervalles de confiance et leurs coefficients de corrélation (tabl. 3). Leur examen montre :

- d'une part, la faible liaison des estimateurs (cf. les coefficients de corrélation) confirmant la nécessité structurelle de ces 3 paramètres; ce qui exprime que chacun des trois organes considérés n'a pas potentiellement le même rôle de réserve (fig. $3 b$ ).

- d'autre part, la bonne sensibilité globale du modèle aux paramètres (cf. les intervalles de confiance) ; il faut noter qu'une variation d'un paramètre $\mathrm{K}_{\mathrm{i}}$ ne peut affecter que l'organe source « $\mathrm{i}$ ) et le ou les organes puis définis par $\mathbf{j}>\mathbf{i}$ (fig. $3 b$ ); ainsi une variation de $K_{3}$ ne peut affecter que les rameaux et les feuilles.

Afin de mieux préciser cette sensibilité, nous avons simulé l'effet d'une variation de chacun des paramètres $K_{i}$ de plus ou moins 2 écart-types, toutes choses égales par ailleurs, en ne faisant varier qu'un seul paramètre à la fois. Cette étude montre que c'est principalement l'organe « $\mathrm{i} »$, source du débit d'azote commandé par $K_{i}$, qui est affecté par cette variation. $P a r$ exemple, une augmentation de $K_{1}$ de 2 écart-types $\left(\mathrm{K}_{1}=0,03:\right.$ tabl. 3$)$ conduit à diminuer la masse d'azote racinaire de $0,068 \mathrm{~g}$ pour $\mathrm{t}=110$ jours (dernière date simulée), soit une diminution d'environ 12 p. 100. Ces $0,068 \mathrm{~g}$ de $\mathrm{N}$ se répartissent entre le tronc, les rameaux et les feuilles dans les proportions de 15, 12 et 73 p. 100, ce qui correspond à une augmentation de la masse d'azote de ces organes inférieure ou égale à 3 p. 100. La plus forte variation relative simulée, pour $\mathrm{t}=110$ jours, concerne les rameaux quand on fait varier $K_{3}:+33$ p. $100\left(K_{3}=0,55:\right.$ tabl. 3$)$ et 20 p. $100\left(K_{3}=1,23:\right.$ tabl. 3$)$ en masse de N. Mais les variations induites au niveau des feuilles sont inférieures à 4 p. 100 .

\section{CONCLUSION}

Le modèle proposé est déterministe dans sa conception. La difficulté tient dans la définition, conceptuelle et expérimentale, de la (des) variable(s) caractérisant les besoins des arbres, besoins qui sont la traduction d'un potentiel de croissance (et de développement) lui-même lié à l'ensemble des conditions environnementales et phytotechniques. Le choix que nous avons fait de représenter les besoins par le " produit final » limite l'utilisation directe qui peut être faite de ce type de modèle à des périodes où il y a concordance entre l'expression de ces besoins et la croissance en matière sèche. Mais on peut imaginer que d'autres indicateurs des besoins puissent être utilisés sans pour cela modifier la structure même du modèle.

Cependant, cette formalisation, certainement imparfaite, du concept de besoin nous a permis de mettre en évidence la logique de répartition de l'azote absorbé dans l'arbre en introduisant le minimum de connaissances a priori nécessaire au fonctionnement du système. Ainsi, il est remarquable de constater que, concernant l'azote du tronc, les phases classiques de diminution puis augmentation des masses ou teneurs, dites de mobilisation et de constitution des réserves, apparaissent comme liées au " programme » de croissance des organes végétatifs. De la même façon, les hypothèses du modèle nous ont permis de reproduire de façon satisfaisante l'ensemble de la dynamique de répartition en masse de l'azote pendant la phase de croissance exponentielle de la matière sèche. Concernant les teneurs, les simulations sont de moins bonne qualité, mais respectent les formes géné- 
rales des courbes déduites des observations expérimentales (HABIB, 1987).

La validation du modèle, c'est-à-dire la confrontation des résultats de simulation à des expérimentations indépendantes de celle ayant servi à l'ajustement des paramètres, fait l'objet d'un $2^{\mathrm{e}}$ article (MONESTIER \&
HABIB, 1987). Dans cet article, seront également présentées l'analyse globale des résidus «mesuresmodèle » et l'utilisation en simulation du fonctionnement du système.

Reçu le 7 août 1986. Accepté le 16 mars 1987.

\section{RÉFÉRENCES BIBLIOGRAPHIQUES}

Bachacou J., Masson J.-P., Millier C., 1981. Manuel de la programmathèque statistique AMANCE 1981. Centre National de Recherches Forestières, INRA, 54280 Champenoux, $516 \mathrm{p}$.

Baldwin J.-P., 1976. Competition for plant nutrients in soil : a theoretical approach. J. agric. Sci. Camb., 87, 341-356.

Baldwin J.-P., Nye P.-H., Tinker P.-B., 1973. Uptake of solutes by multiple root systems from soil. III. A model for calculating the solute uptake by a randomly dispersed root system developing in a finite volume of soil. Plant Soil, 38, 621-635.

Barnes A., Greenwood D.-J., Cleaver T.-J., 1976. A dynamic model for the effects of potassium and nitrogen fertilizers on the growth and nutrient uptake of crops. J. agric. Sci. Camb., 86, 225244.

Bhat K. K. S., 1982. Nutrient inflow into apple roots. II. Nitrate uptake rates measured on intact roots of mature trees under field conditions. Plant Cell Environ., 5, 461-469.

Bhat K. K. S., Brereton A. J., Nye P.-H., 1979. The possibility of predicting solute uptake and plant growth response from independently measured soil and plant characteristics. VII. A comparison of the growth and nitrate uptake of rape grown in similar nitrate concentration in solution or in soil solution. Plant Soil, 53, 193-201.

Bhat K. K. S., Nye P.-H., 1980. Plant attributes involved in the nitrate absorbing power of roots. J. Sci. Food Agric., 31 (8), 851852.

Bresler E., 1973. Simultaneous transport of solutes and water under transient, unsaturated flow conditions. Water resour. Res., 9, 975 . 986.

Burns I. G., 1980. Influence of the spatial distribution of nitrate on the uptake of $\mathrm{N}$ by plants : a review and a model for rooting depth. J. Soil Sci., 35, 155-173.

Chevalier F., Lefevre C., 1984. Systèmes et modèles. Une introduction à la méthode des indicateurs. Ed. du CNRS (Paris), $250 \mathrm{p}$.
Grignon C., Touraine B., 1981. Les fonctions de transport de la racine. I. Les fonctions de prélèvement. Physiol. vég., 19 (3), 443-462.

Habib R., 1984. La formation des réserves azotées chez les arbres fruitiers. Revue bibliographique. Fruits, 39 (10), 623-635.

Habib R., 1987. Effet de la disponibilité en nitrate sur le comportement de jeunes pêchers en culture hydroponique. Agronomie, 7 (2), 101-109.

Lassales J..P., Thellier M., 1974. Modèles théoriques utilisables pour la description des échanges cellulaires. Physiol. vég., 12, 665688 .

Lebreton J. D., Millier C., 1982. Modèles dynamiques déterministes en biologie. Masson ed. (Paris), $208 \mathrm{p}$.

Monestiez P., Habib R., 1987. Modélisation de la dynamique de répartition de l'azote chez un jeune arbre fruitier pendant la phase de croissance exponentielle. II. Validation. Analyse de l'erreur. Simulation. Agronomie, 7 (7).

Nye P.-H., 1979. In The soil-root interface. Soil properties controlling the supply of nutrients to the root surface. Ed. J.-L. Harvey and R. S. Russel Acad. Press (London), 39-49.

Nye P.-H., Marriot F. H. C., 1969. A theoretical study of the distribution of substances around roots resulting from simultaneous diffusion and mass flow. Plant Soil, 30 (3), 459-472.

Stoer J., Bulirsch R., 1980. Introduction to numerical analysis. Springer-Verlag ed. (New York), $609 \mathrm{p}$.

Therios I. N., Weinbaum S. A., Carlson R. M., 1979. Nitrate compensation points of several plum clones and relationship to nitrate uptake effectiveness. J. amer. Soc. hortic. Sci., 104 (6), 768-770.

Titus J. S., Kang S. M., 1982. Nitrogen metabolism, translocation and recycling in apple trees. Hort. Rev., 4, 204-246.

Tromp J., 1983. Nutrient reserves in fruit trees, in particular carbohydrates and nitrogen. Plant Soil, 71, 401-414. 